\title{
Endoscopic management of Lemmel syndrome: a rare, benign cause of obstructive jaundice
}

\author{
G P U P de Silva ${ }^{1}$, S L Delwatta ${ }^{2}$, R G M S Nandasena ${ }^{2}$, I W M P Wanigasooriya ${ }^{1}$, A A Pathirana \\ ${ }^{1}$ Colombo South Teaching Hospital, Kalubowila, Sri Lanka \\ ${ }^{2}$ Faculty of Medical Sciences, University of Sri Jayewardenepura, Sri Lanka
}

Keywords: Periampullary; duodenal diverticula; obstructive jaundice; Lemmel syndrome

\section{Introduction}

In 1934, the German physician Gerhard Lemmel described a condition where periampullary duodenal diverticuli caused obstructive jaundice in the absence of cholelithiasis, biliary strictures or neoplasms [1]. This entity is still less familiar due to the rarity of the condition and the scarcity of literature.

As it can mimic sinister pathology in the distal biliary or periampullary region, accurate diagnosis is imperative to minimize mismanagement.

\section{Case presentation}

\section{Patient 1}

An 81-year-old male developed progressive obstructive jaundice, nausea and poor appetite. He denied fever, weight loss and a history of cholelithiasis. Direct hyperbilirubinemia with elevated alkaline phosphatase (ALP) and $\mathrm{y}$ glutamyl transferase ( $\mathrm{Y}$ GT) levels was observed. Aspartate aminotransferase (AST) and alanine transferase (ALT) were within the normal range. Ultrasonography revealed dilatation of gallbladder, intra and extrahepatic bile ducts. Contrastenhanced computed tomography (CECT) abdomen and magnetic resonance cholangiopancreatogram (MRCP) demonstrated a smooth narrowing of the distal bile duct with proximal biliary dilatation and a periampullary diverticulum (Figure 1). The major papilla was located in between two large periampullary diverticula during the endoscopic retrograde cholangiopancreatogram (ERCP) without choledocholithiasis or biliary stricture. A plastic biliary stent was placed following limited selective biliary sphincterotomy. The patient became asymptomatic with improved liver functions. A repeat ERCP was performed after 8 weeks. Biliary sphincterotomy was extended and the stent was removed.

Correspondence: G. P. U. P. de Silva

E-mail: upulonline@gmail.com

(iDhttps://orcid.org/0000-0003-2508-3765

Received: 02-07-2021 Accepted: 01-11-2021

DOI: http://doi.org/10.4038/sljs.v39i3.8858

The Sri Lanka Journal of Surgery 2021; 39(3): 97-101

\section{Patient 2}

A 72-year-old female was admitted to the surgical casualty with septic shock due to ascending cholangitis. She denied previous episodes of biliary colic or obstructive jaundice. She had a rising level of direct hyperbilirubinemia, ALP, y GT and marginally elevated AST and ALT. Ultrasonography revealed intra- and extrahepatic duct dilatation, and a stone in the distal common bile duct (CBD). Urgent ERCP demonstrated two periampullary diverticula causing distal CBD narrowing and a stone proximal to it (Figure $2 \mathrm{a}$ and $2 \mathrm{~b}$ ). The patient recovered following biliary decompression with a plastic biliary stent. Removal of the stent, a selective biliary sphincterotomy and balloon extraction of CBD stone was performed in 8 weeks (Figure 2c).

\section{Patient 3}

An 80-year-old female developed right hypochondrial pain and poor appetite for 3 months in the absence of obstructive jaundice. Her abdominal examination was unremarkable. Total bilirubin, ALP, and y GT were slightly elevated. Ultrasonography demonstrated a distended gallbladder with intra and extrahepatic duct dilatation. MRCP indicated distal biliary stenosis causing proximal dilatation. ERCP demonstrated three periampullary diverticula causing distal biliary obstruction in the absence of a biliary stricture. A plastic biliary stent was placed following a selective biliary sphincterotomy. Repeat ERCP after 8 weeks revealed a proximal stent migration which required an extension of biliary sphincterotomy and strenuous removal using a stone retrieval basket (Figure 3).

\section{Patient 4}

A 74-year-old female presented with progressive jaundice, epigastric pain, poor appetite and intermittent fever without chills for 2 weeks. Her abdominal examination was unremarkable. Direct hyperbilirubinemia and elevated ALP and $\mathrm{\gamma}$ GT were noted. Ultrasonography demonstrated a distended gallbladder with intra and extrahepatic duct dilatation, MRCP revealed distal biliary stenosis causing proximal dilatation and few CBD stones. ERCP showed that the major papilla was located in the inner lip of a large duodenal diverticulum. Selective biliary cannulation and placement of a plastic biliary stent were performed with difficulty (Figure 4a and 4b). Sphincterotomy or stone 

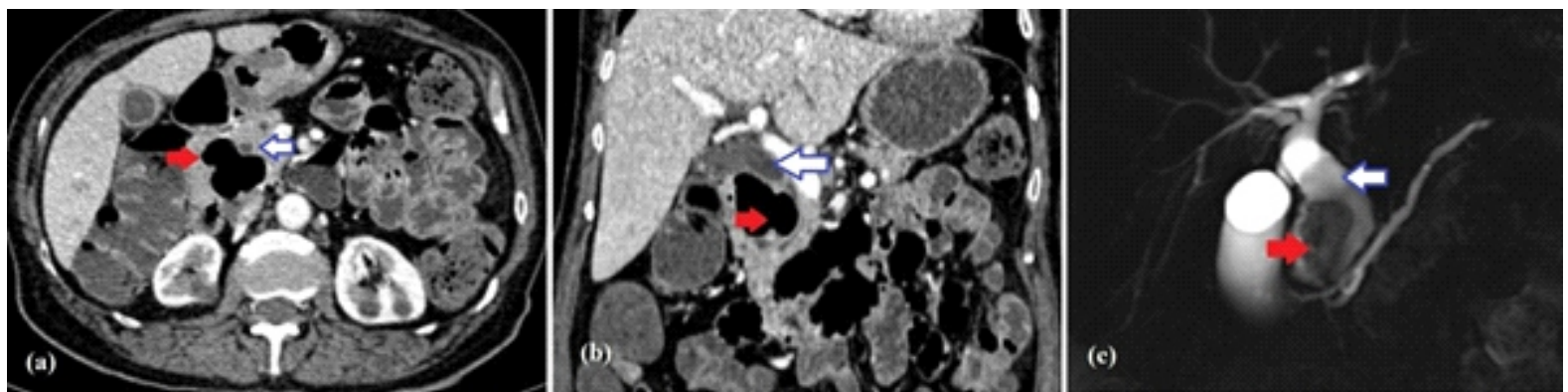

Figure 1: A large periampullary duodenal diverticulum (red arrow) causing dilatation of the proximal biliary ducts (white arrow). (a) Axial view of a computed tomography. (b) Coronal view of a computed tomography. (c) Magnetic resonance cholangiopancreatography.

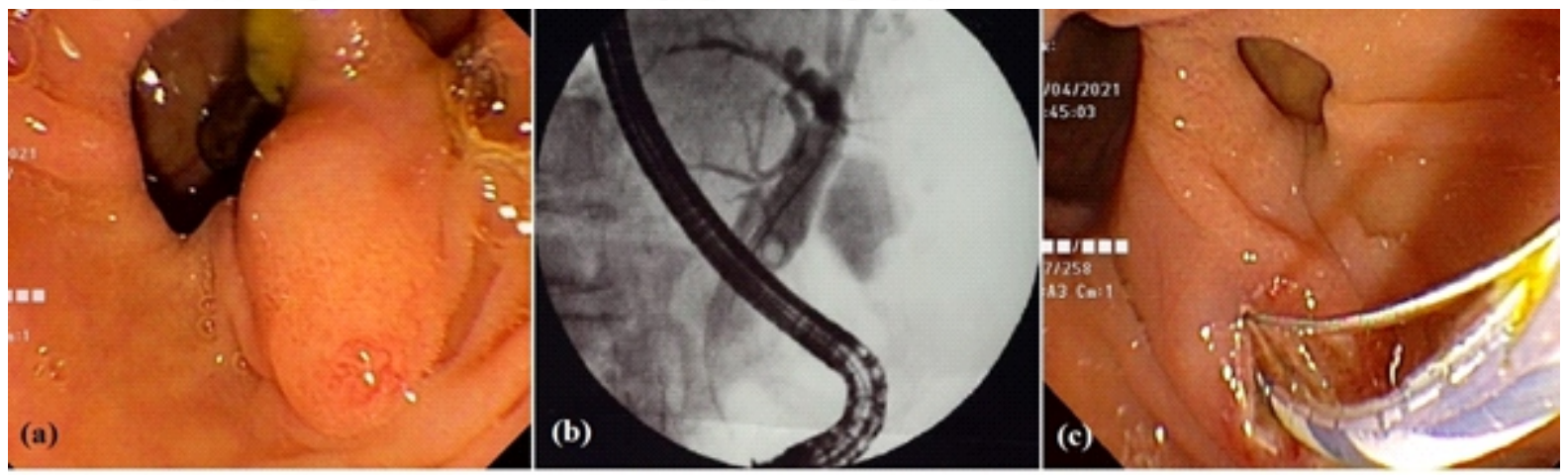

Figure 2: (a) Side viewing endoscopy showed the major papilla lying in between two large periampullary diverticula. (b) Endoscopic retrograde cholangiogram shows dilated intra- and extra hepatic bile ducts with a stone in the common bile duct proximal to the distal stenosis. (c) Selective biliary sphincterotomy.

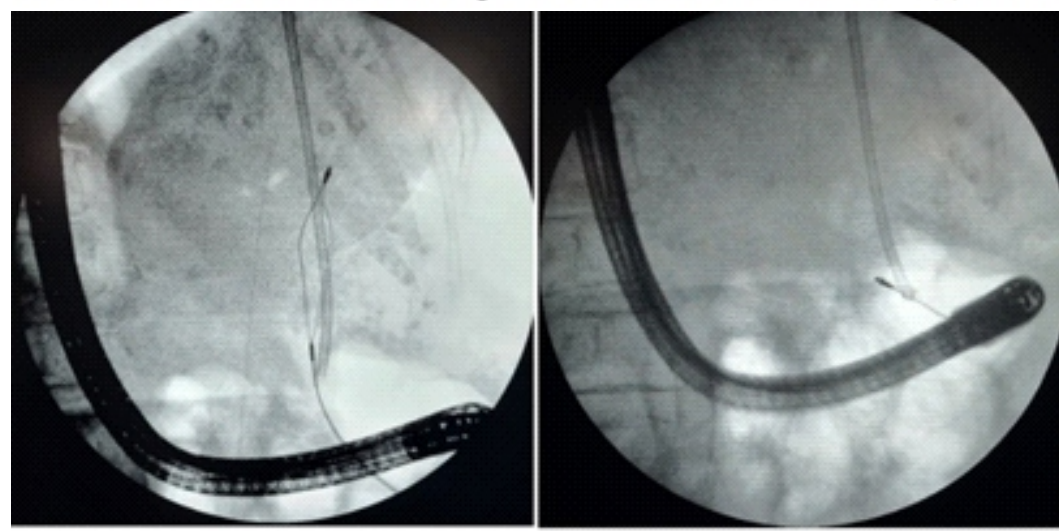

Figure 3: Proximally migrated stent was retrieved using a stone retrieval basket.
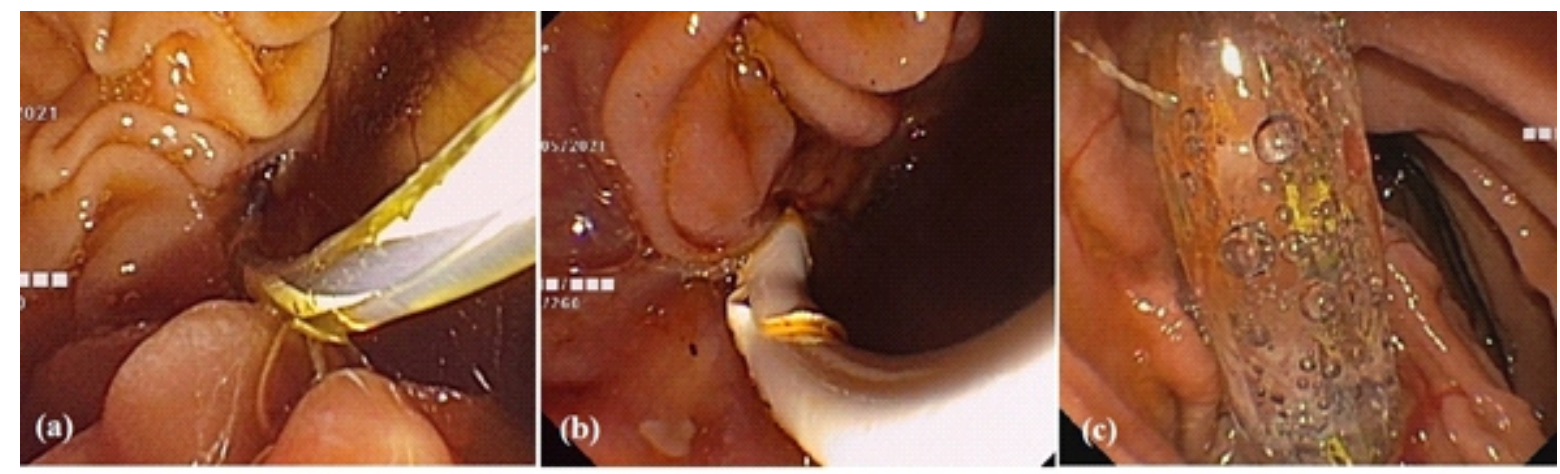

Figure 4: (a) difficult selective biliary cannulation as the major papilla located in the inner lip of a large duodenal diverticulum. (b) Placement of a plastic biliary stent. (c) Sphincteroplasty was performed subsequently to facilitate balloon extraction of common bile duct stones. 
extraction was not attempted during the initial ERCP. Sphincteroplasty and balloon extraction of stones was performed during the repeat ERCP (Figure 4c).

\section{Discussion}

The duodenum is the commonest location in the small intestine for pulsion type pseudodiverticuli which consist of mucosa, submucosa and serosa protruding through the muscularis propria. Increased intraluminal pressure associated with abnormal intestinal contractions or localized inflammation is presumed to cause the herniation through the weak intestinal wall where blood vessels penetrate [1].

The incidence of duodenal diverticula ranges from $7.5-27 \%$ based on endoscopic ultrasound and ERCP studies. While many are asymptomatic, some develop complications including haemorrhage, obstruction, inflammation, and perforation. The majority $(70 \%)$ are in the second part of the duodenum along its medial border and may produce pancreaticobiliary manifestations [1].

The eponym Lemmel syndrome is referred to when a periampullary diverticulum exerts mechanical compression on the common bile duct causing obstructive jaundice. Following endoscopic manometry studies, Tomita et al. suggested that sphincter of Oddi dysfunction also contributes to the condition [2]. Other proposed mechanisms include chronic fibrosis of the papilla (papillitis Chronica fibrosa) and distention of periampullary diverticulum by bezoar or enterolith [3]. However, macroscopic fibrotic changes of the major papilla or significant intraluminal collections causing diverticular distension were not observed in our series [Table $1]$.

Lemmel syndrome is common among older patients with a female predominance as we observed in this series $[3,4]$. Progressive or intermittent jaundice associated with variable symptoms (epigastric pain, abdominal fullness, loss of appetite) has been documented in the previous reports. A subset of patients can present with ascending cholangitis as observed in 'patient 2'. Common biochemical findings include direct hyperbilirubinemia, elevated ALP and 8 GT with normal or marginally elevated AST and ALT levels. [4]. Normal C-reactive protein and leucocyte levels were observed in the majority in this series except for the patient with ascending cholangitis.

The literature describes the image appearance of periampullary diverticula as rounded sacs containing air, along the medial wall of the second part of the duodenum. However, differentiation of the diverticula from walled-off necrosis, pancreatic pseudocyst or abscess may be challenging [3 - 5]. Frauenfelder et al. recommend the use of CECT with oral contrast to demonstrate communication with the duodenal lumen, estimate its size, and reveal extrinsic compression on the bile duct. Administration of intravenous contrast demonstrates homogeneous enhancement of the diverticular wall in the absence of a mass differentiating from a neoplasm or inflammatory lesions [4]. MRCP has a sensitivity of $81.2 \%$ and specificity of $98.6 \%$ to detect a single periampullary diverticulum and helps further evaluation of aetiology for the biliary obstruction [5]. In our series, a combination of imaging modalities was performed to exclude malignant pathologies considering the old age at presentation, distended gallbladder with intra- and extrahepatic biliary duct dilatation detected in ultrasonography. Image (CECT or MRCP) evidence of periampullary diverticula was observed only in 'patient 1'. The diagnoses of Lemmel syndrome were made during multidisciplinary team discussions when the imaging failed to show cholelithiasis, masses or strictures as the cause for distal biliary obstruction in the presence of periampullary diverticula observed in the side-viewing endoscopy.

Management of Lemmel syndrome can be conservative, endoscopic, and surgical. Conservative management is suitable for patients who have incidentally detected periampullary diverticula with minimal biliary obstruction in the absence of hyperbilirubinemia. Endoscopic management is commonly described in the literature, whereas surgical management (diverticulectomy or biliodigestive anastomosis) is performed rarely $[3,4]$.

All patients in this series were managed successfully with endotherapy. Performing selective biliary cannulation and biliary sphincterotomy in the presence of a diverticulum was technically demanding. Plastic biliary stents were inserted following the sphincterotomy. In one patient, stenting was performed without a sphincterotomy as the major papilla was located on the inner lip of a large diverticulum. Two patients had developed common bile duct stones due to distal obstruction. We avoided stone extraction during the first ERCP considering the risk of perforation.

All the patients underwent a second ERCP after 2 months. Balloon extraction of the stones was done with ease as the stones had become soft in the presence of the stent. Once a clear biliary tree was confirmed with an occlusion cholangiogram and the presence of free bile flow, re-stenting was not required in these patients.

The risk of complications related to ERCP including perforation, acute pancreatitis, and cholangitis are higher in these patients [4]. Except for the stent migration, none of our patients developed sinister complications following ERCP. They are being followed up at the surgical clinic 3 monthly with liver functions. 
Table 1. Summary of demographic data, clinical presentation, investigations, and treatments

\begin{tabular}{|c|c|c|c|c|}
\hline & Patient 1 & Patient 2 & Patient 3 & Patient 4 \\
\hline Age (years) & 81 & 72 & 80 & 74 \\
\hline Gender & Male & Female & Female & Female \\
\hline Comorbidities & Hypertension & Diabetes mellitus & Hypertension & Not evaluated \\
\hline Presenting complaints & \begin{tabular}{l|} 
Jaundice \\
Loss of appetite
\end{tabular} & $\begin{array}{l}\text { Jaundice } \\
\text { Fever } \\
\text { Epigastric pain }\end{array}$ & $\begin{array}{l}\text { Right hypochondrial pain } \\
\text { Loss of appetite }\end{array}$ & $\begin{array}{l}\text { Jaundice } \\
\text { Epigastric pain }\end{array}$ \\
\hline Total bilirubin ( $\mu \mathrm{mol} / \mathrm{l})$ & 78 & 153 & 24 & 83 \\
\hline Direct bilirubin ( $\mu \mathrm{mol} / \mathrm{l})$ & 56 & 81 & 15 & 33 \\
\hline $\operatorname{ALP}(U / I)$ & 332 & 497 & 287 & 304 \\
\hline y GT (U/I) & 91 & 516 & 43 & 105 \\
\hline AST & 42 & 87 & NA & NA \\
\hline ALT & 34 & 92 & NA & NA \\
\hline \multicolumn{5}{|l|}{ Ultrasonography } \\
\hline Diameter of CBD (mm) & 14 & 14 & 16 & 18 \\
\hline Intrahepatic ducts & Dilated & Dilated & Dilated & Dilated \\
\hline Gallbladder & Distended & Not distended & Distended & Distended \\
\hline CECT and MRCP & $\begin{array}{l}\text { Distal biliary } \\
\text { stenosis, } \\
\text { periampullary } \\
\text { diverticulum }\end{array}$ & Not performed & Distal biliary stenosis & Distal biliary stenosis \\
\hline \multicolumn{5}{|l|}{ ERCP } \\
\hline Findings & $\begin{array}{l}2 \text { periampullary } \\
\text { diverticula }\end{array}$ & $\begin{array}{l}2 \text { periampullary } \\
\text { diverticula, a CBD stone }\end{array}$ & $\begin{array}{l}3 \text { periampullary diverticula, } \\
\text { a CBD stone }\end{array}$ & $\begin{array}{l}\text { Single large periampullary } \\
\text { diverticulum }\end{array}$ \\
\hline Treatments & $\begin{array}{l}\text { Biliary } \\
\text { sphincterotomy } \\
\text { and stenting }\end{array}$ & $\begin{array}{l}\text { Biliary sphincterotomy, } \\
\text { stone extraction and } \\
\text { stenting }\end{array}$ & $\begin{array}{l}\text { Biliary sphincterotomy and } \\
\text { stenting }\end{array}$ & $\begin{array}{l}\text { Biliary sphincterotomy, } \\
\text { stone extraction and } \\
\text { stenting }\end{array}$ \\
\hline Complications & & & Proximal migration of stent & \\
\hline
\end{tabular}

ALP, alkaline phosphatase; 8 GT, gamma glutamyl transferase; AST, aspartate aminotransferase; ALT, alanine transferase; CECT, contrast enhanced computed tomography; MRCP, magnetic resonance cholangiopancreatography; ERCP, endoscopic retrograde cholangiopancreatography; $N A$, not available

\section{Conclusion}

Lemmel syndrome is a rare cause of distal biliary obstruction by periampullary diverticula commonly seen among the elderly. It should be considered in the differential diagnosis of obstructive jaundice in the absence of cholelithiasis, tumour, inflammatory or iatrogenic strictures. Endotherapy with biliary sphincterotomy and stenting is successful in most patients.

All authors disclose no conflict of interest. The study was conducted in accordance with the ethical standards of the relevant institutional or national ethics committee and the Helsinki Declaration of 1975, as revised in 2000

\section{References}

1. Rangan V, Lamont JT. Small Bowel Diverticulosis: Pathogenesis, Clinical Management, and New Concepts. Curr Gastroenterol Rep. 2020 Jan 15;22(1):4. doi: 10.1007/s11894-019-0741-2. PMID: 31940112.
2. Tomita R, Tanjoh K. Endoscopic manometry of the sphincter of Oddi in patients with Lemmel's syndrome. Surg Today. 1998;28(3):258-61. doi: 10.1007/s005950050117. PMID: 9548305 .

3. Bernshteyn M, Rao S, Sharma A, Masood U, Manocha D. Lemmel's Syndrome: Usual Presentation of an Unusual Diagnosis. Cureus. 2020 Apr 16;12(4):e7698.

doi: 10.7759/cureus.7698. PMID: 32431977; PMCID: PMC7233497.

4. Frauenfelder G, Maraziti A, Ciccone V, Maraziti G, Caleo O, Giurazza F, Zobel BB, Carbone M. Computed Tomography Imaging in Lemmel Syndrome: A Report of Two Cases. J Clin Imaging Sci. 2019 May 24;9:23. doi: 10.25259/JCIS-17-2019. PMID: 31448174; PMCID: PMC6702893.

5. Getsov P, Mitova-Siminkovitch S, Dineva S, Vladimirov B. Diagnostic accuracy of MRCP in evaluation of periampullary duodenal diverticula - prospective study. Trakia Journal of Sciences. 2020;18(2):105-110. doi: 10.15547/tjs.2020.02.003. 


\section{Learning Points:}

- Lemmel syndrome is a rare condition presented with obstructive jaundice caused by periampullary duodenal diverticulum in the absence of cholelithiasis, stricture or neoplasm.

- Lemmel syndrome is usually seen among elderly population.

- Endotherapy with biliary sphincterotomy and stenting is successful in most patients. 